\title{
Improved Noise Isolation Design of UHF Mobile Radio Frequency Identification Reader System in Package
}

\author{
Yujeong Shim, Jongbae Park, Youngwon Kim, Kyoungchoul Koo and Joungho Kim \\ Terahertz Interconnection and Package Laboratory, \\ Dept. of EECS,KAIST,373-1 Gusung,Yusong,Daejeon 305-701,Korea \\ Phone) +82-42-879-9873, Fax)+82-42-869-8058, E-mail) ssimu@eeinfo.kaist.ac.kr;teralab@ee.kaist.ac.kr
}

\begin{abstract}
Recently, one of the most frequently mentioned keyword is "Ubiquitous", which shows that radio frequency identification (RFID) becomes important and its demands are extremely expanding in the market. As mobile applications are widely spreaded, the importance for the RFID expands not only to the stationary reader systems but also the mobile readers.

As the size of the mobile application with multi-function decreases, the most considerable factors of the RFID reader used in mobile applications are size and power consumption. We have designed two chips including a digital circuit, a receiver, and a transmitter. Furthurmore, we have integrated those designed chips in a single package whose dimension is $23 \mathrm{~mm}$ by $23 \mathrm{~mm}$ with low power consumption (1 watt). In this paper, we propose and verify design of chips and package for the mobile RFID reader.
\end{abstract}

\section{Introduction}

In the past days, a revolution in identification systems was triggered by barcode labels, but data storage in a silicon chip has been considered as a novel optimal solution. And a number of companies has involved in the development and sale of radio frequency identification (RFID) systems whose gravity is increasing. Recently, one of the most frequently mentioned keyword is "Ubiquitous", which shows that the portable RFID application becomes important and its demands are extremely expanding[1].

13.56 MHz RFID such as a traffic card, an ID card, and a credit card is one of the most popular system in these days. However, we expect that the demand for the ultra high frequency (UHF) identification system will increase based on its long identification range, small size, low cost of antenna, and fast data processing. And the arrival of "Ubiquitous" age has increased the importance of mobile RFID rather than stationary RFID.

We have designed an RFID reader for portable applications that has so low power consumption (1 watt) and small size $(23 \times 23 \mathrm{~mm})$ as to be suitable for the mobile phones and portable equipments. Since system-in-package ( $\mathrm{SiP}$ ) of the UHF-band RFID includes noise-sensitive analog circuitries, considerations of signal integrity and power integrity makes it possible to be a high performance system

\section{Mobile RFID reader and Designed Chips and SiP}

System block diagram is shown in figure 1 . We have designed two chips including a digital circuit, a receiver, and a transmitter. Furthurmore, we have integrated those designed chips in a SiP with two main targets; low power consumption and small size. As shown in figure 1, the designed RFID reader has two parts including digital and analog part. The digital part consists of a micro controller unit (MCU) and a digital base-band modem, while the analog part is composed of a receiver and a transmitter. Usually, RFID reader has a high speed clocked(160MHz) DSP or ARM based CPU, but we design a $16 \mathrm{MHz}$ clocked 16-bit reduced-instruction-set-computer (RISC) to acheave a low power consumption system. Therefore, we could reduce computing power to 10 percents.

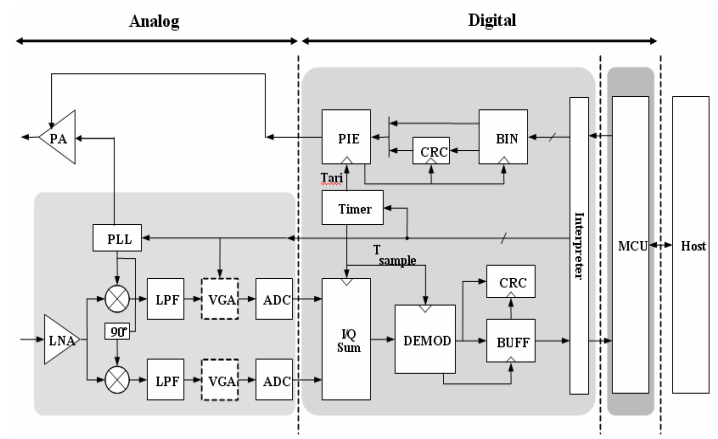

Figure 1. System block diagram of the designed RFID reader

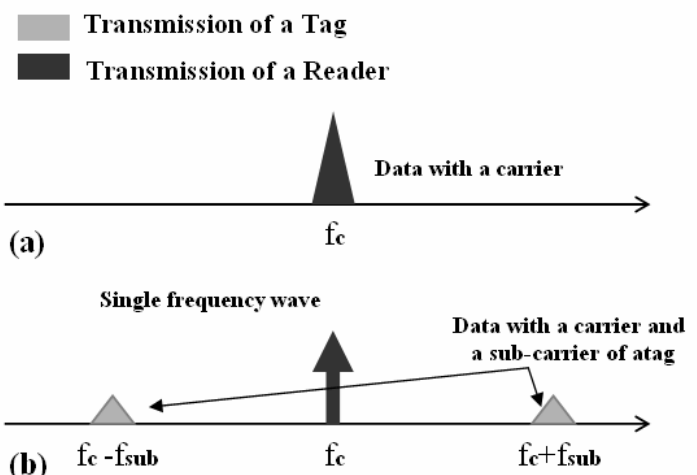

Figure 2. Spectrum in air condition

(a) Spectrum of reader transmission to a tag

(b) Spectrum when a tag responses to a reader

If the system is implemented as one chip-based SiP, the system size becomes smaller. However, in our design, two chips-based $\mathrm{SiP}$ is chosen for the noise coupling isolation between the transmitter and the receiver. Because an UHF RFID tag does not use battery, it receives and transmits data with power from the reader. When a tag transmits data to a reader, the reader transmit a single frequency continuous 
wave to supply the power to a tag. The tag modulates data with a sub-carrier to avoid interference between the tag and reader. Figure 2(a) shows the spectrum measured when the reader transmits data to the tag, and figure $2(\mathrm{~b})$ shows the spectrum measured when the tag transmits data to the reader. But the sub-carrier frequency is only several hundreds $\mathrm{kHz}$ and the transmitted power is about 3 to 20 times higher than the received power. Thus, it is hard to avoid interference between the reader and the tag, which degrades system performance.

There are two kinds of noise coupling. First, since mobile RFID reader has only one antenna to transmit and receive signals, there is a coupling between $\mathrm{Rx}$ and $\mathrm{Tx}$ signal. Second, there is a substrate coupling between receiver and transmitter in the case that they are designed in a single chip. We select a circulator which has $-35 \mathrm{~dB}$ isolation level to reduce the leakage of power from transmitter to receiver and separate the transmitter with the receiver to suppress the substrate coupling.

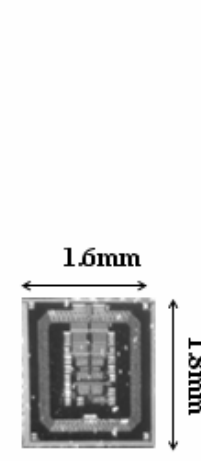

(a)

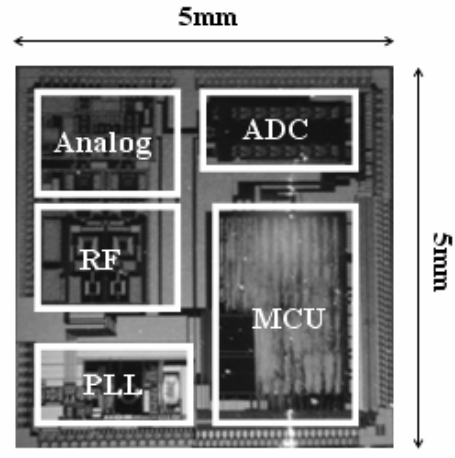

(b)
Figure 3. (a) Transmitter chip with power amplifier used direct ASK modulation. (b) Digital and receiver chip including MCU, DBM, PLL, ADC, analog and RF circuits.

Figure 3 shows two designed chips. Figure 3.(a) is the direct modulated power amplifier to modulate data with Amplitude Shift Keying (ASK) like a mixer. Figure 3.(b) shows the chip which consists of DBM, MCU, SRAM, PLL, analog circuits and RF circuits. As shown in Figure 4, the designed SiP has four layers and 456 balls. There are ten power planes and five ground planes.

\section{Effect of Noise Coupling on Circuits}

It is described in the previous chapter that we design the two-chips-based SiP to improve the isolation between TX and RX. In this chapter, we will compare two-chips-based $\mathrm{SiP}$ with one-chip-based $\mathrm{SiP}$ and show the effect of noise coupling on other circuits.
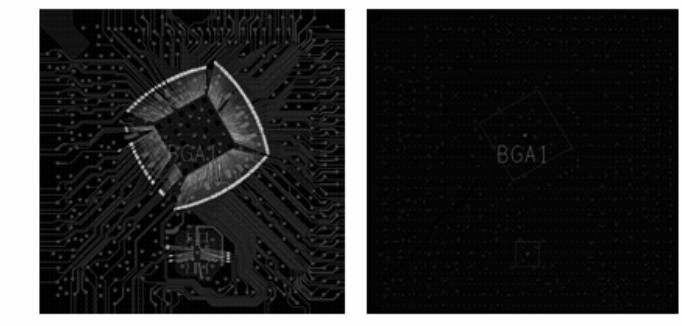

$1^{\text {st }}$ plane : signal \& bonding wire $\quad 2^{\text {nd }}$ plane : ground

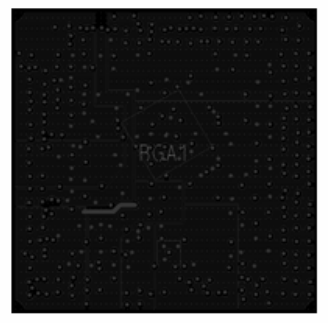

$3^{\text {rd }}$ plane : power

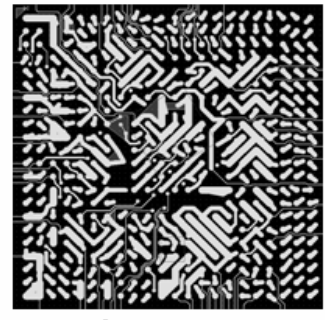

$4^{\text {th }}$ plane : ball
Figure 4. Layout of the designed SiP which has four layers stack up.

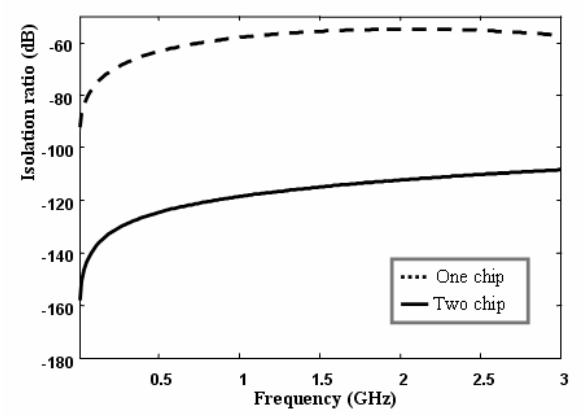

(a)

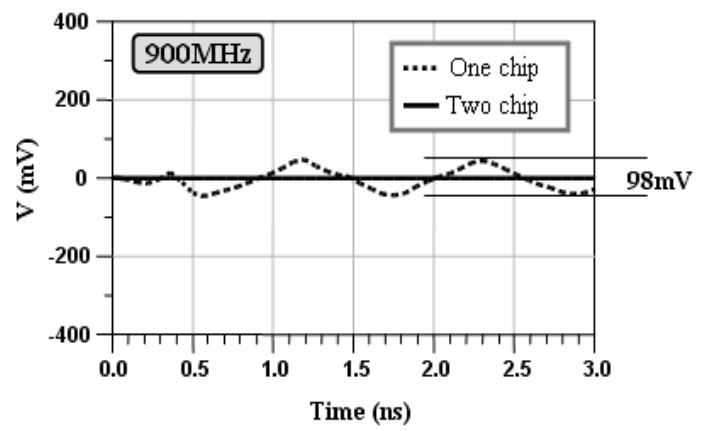

(b)

Figure 5.(a) Noise coupling ratio through substrate in the frequency domain, up to $3 \mathrm{GHz}$, (b) in the time domain for one-chip-based SiP and two-chips-based SiP.

Figure 6(a) shows the graph for the noise coupling ratio through signal line. The solid line and the dashed line show the ratio of the designed $\mathrm{SiP}$ and the single-chip SiP, 
respectively. It is understood that the noise coupling through signal line causes distortion for other signals as shown in figure $6(\mathrm{~b})$ and (c).

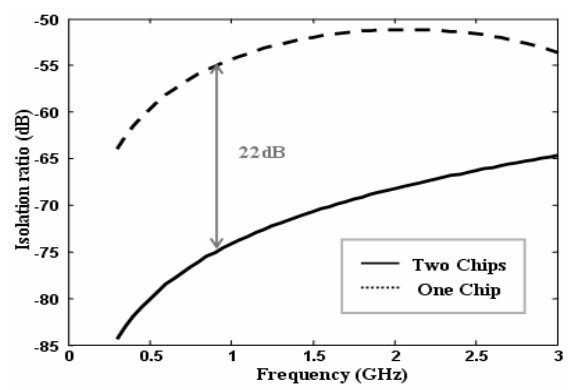

(a)

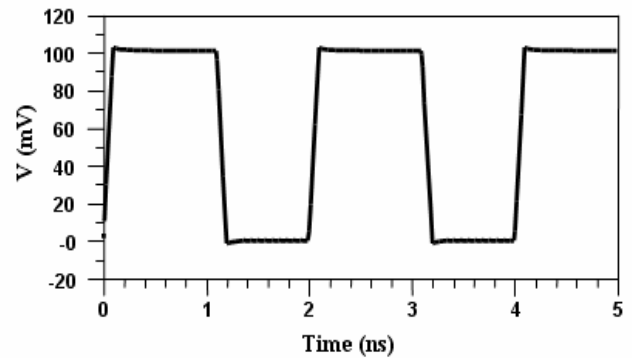

(b)

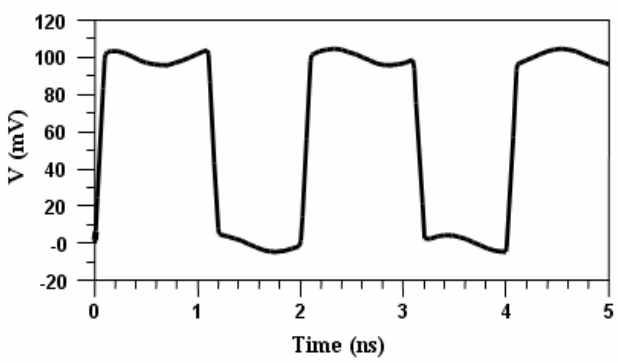

(c)

Figure 6. (a) Isolation ratio of signal line coupling, (b) Signal of seperated chip SiP, (c) Signal of one chip SiP

The coupled signals affect other circuits. Noise coupling through signal and substrate distorts output signals inducing DC offset and making the gross signals as shown Figure 7. Figure 7(a) shows spectrum for the output signal of the designed mixer when there exists the substrate noise coupling. If $500 \mathrm{kHz}$ data are modulated with $900 \mathrm{MHz}$ using the mixer, the output signals consist of $500 \mathrm{kHz}$ and $1800.5 \mathrm{MHz}$ signals [figure 7(a)]. But if there is a noise coupling, an unwanted $934.5 \mathrm{MHz}$ noise signal is added to the $500 \mathrm{KHz}$ output signal of the mixer, as shown in figure 7(b). Furthuremore, the coupled noise is mixed with the input signal of the mixer. Eq. (1) is the equation of the output signals of the mixer without coupling noise and Eq. (2) is that with coupling noise. There is a difference between Eq. 1 and Eq. 2 for the DC values. The DC offset is generated when a system uses a direct conversion receiver. We chose the direct conversion method to reduce the circuit complexity. In this case, a coupled noise are mixed with a LO signal and a received signal [4]. And it generates the unexpected DC offset. After all, self-mixing with substrate coupling noise induces DC offset, and it degrades identifying performance of the system

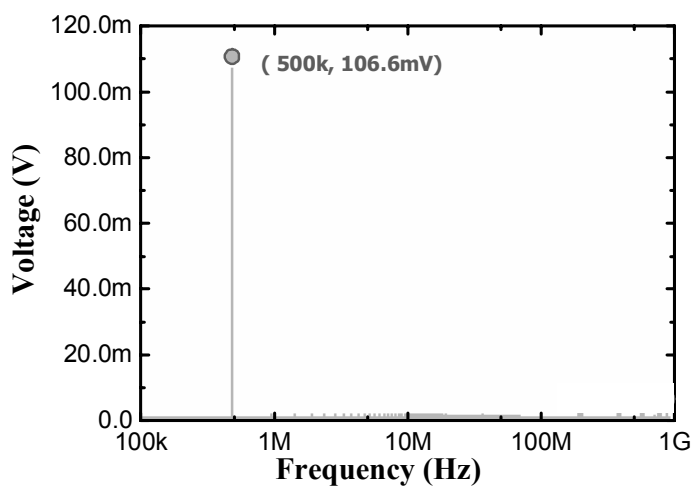

(a)

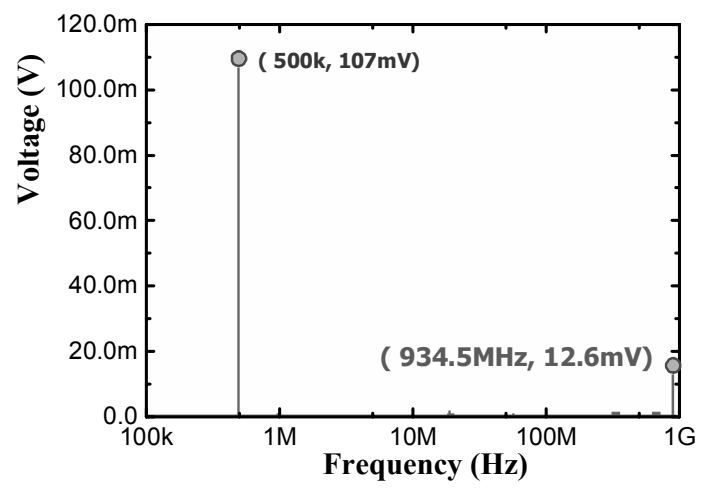

(b)

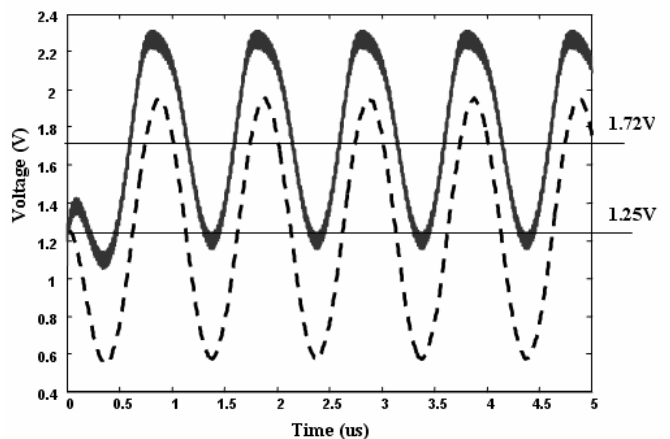

(c)

Figure 7. effect of coupling noise on the circuits (a) the fft result of a mixer output with no noise, (b) the fft result of a mixer output with coupling noise, (c) output signal of opamp.

Figure $7(\mathrm{~b})$ indicates the output signals of operational amplifier. In Figure 7(b), the dashed line and the solid line represent the output signal without and with power/ground 
noise, respectively. Signal and power/ground noise coupling induce a distortion of output signals [Eq (3)], increasing an output offset and reducing voltage gain.

$$
\begin{aligned}
V o= & A \cos \omega t \times B \cos \omega t \\
& =A B \frac{1+2 \cos \omega t}{2} \\
& =\frac{A B}{2}+A B \cos \omega t \\
V o= & (A \cos \omega t+C \cos \omega t) \times B \cos \omega t \\
& =A B \frac{1+2 \cos \omega t}{2}+B C \frac{1+2 \cos \omega t}{2} \\
& =\frac{B(A+C)}{2}+B(A+C) \cos \omega t \\
V_{\text {off }}= & \frac{i_{d}}{g_{m}}
\end{aligned}
$$

\section{Suppression of Noise Coupling through Signal and Substrate}

On the basis of previous results, we need to improve the noise isolation for the SiP design. In previous chapter, we separated the transmitter with the receiver to reduce the noise coupling through the signal and the substrate since the power of the transmitter is much larger than that of the receiver. In this chapter, several noise isolation techniques will be proposed and verified for the designed UHF mobile RFID reader $\mathrm{SiP}$, using the 3D full wave simulation.

As the power amplifier of a direct modulation formula consumes far larger current than other blocks, it generates a significant power/ground noise. Therefore, the power/ground plane of the power amplifier chip should be separated from one of another chip.

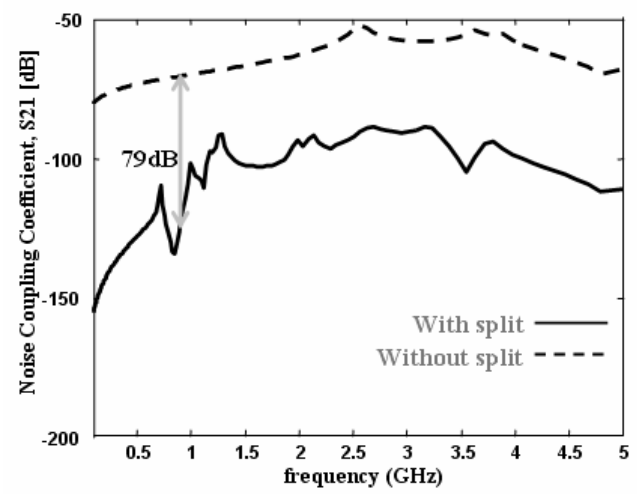

Figure 8. Noise coupling coefficient of power/ground Plane. At $900 \mathrm{MHz}$, the isolation ratio with split is improved as $79 \mathrm{~dB}$ as without split.
Figure 8 represents that the split plane reduces the power/ground noise coupling by $80 \mathrm{~dB}$ at $900 \mathrm{MHz}$ between power amplifier and other chips.

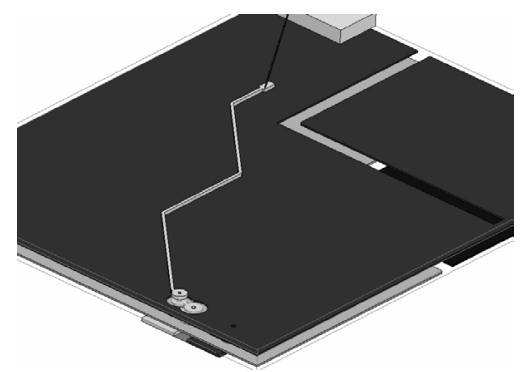

(a)

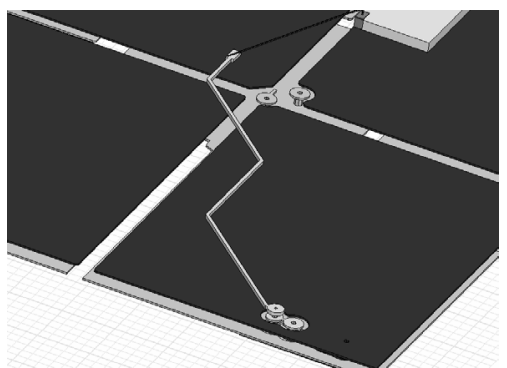

(b)

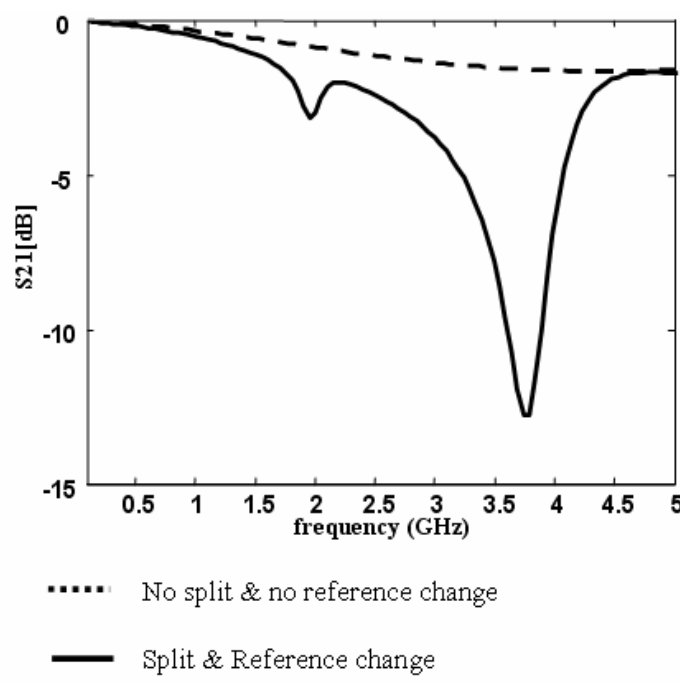

(c)

Figure 9. comparison of insertion loss of a signal line with split plane and reference change, and without split plane and reference change (a) signal line without split plane and reference change, (b) signal line with split plane and reference change, (c) Insertion loss about a worst case line

However, signal integrity is worsen by a split on plane. A gap between planes makes a return current path long, which increases inductance. The inductance and the 
capacitance of the gap make a new resonance frequency which degrades the transmission characteristic of a signal. However, as shown in figure 9, there is negligible effect of $\mathrm{P} / \mathrm{G}$ plane split on signal integrity at $900 \mathrm{MHz}(-0.44 \mathrm{~dB}$ insertion loss).

\section{Conclusions}

In this paper, to minimize the noise coupling between a transmitter and a receiver, the digital and receiver chip, the TX chip that separates with the RX chip and two chipsbased RFID SiP have been proposed. Therefore, to improve noise isolation characteristic, several noise isolation techniques has been shown. As a result, interference between $\mathrm{Tx}$ and $\mathrm{Rx}$ signals and the power/ground noise coupling of transmitter including direct modulation power amplifier have been reduced. And the mobile RFID reader SiP which has small size and low power consumption has been implemented.

\section{Acknowledgments}

The authors appreciate all participants' pains of the chip design. Also thanks to Joungho Shim, Kicheol Bae, Jinhan Kim and Amkor to manufacture the SiP

\section{References}

1. Klaus Finkenzeller. RFID Handbook : Fundamentals and applications in Contactless Smart Cards and Identification, Jogn Wiley \& Sons,Ltd

2. Nadeem Raza, Viv Bradshaw, Matthew Hague, Microlise Systems Integration Limited, "Applications of RFID Technology", IEE Colloquium on 25 Oct. 1999 Page(s):1/1 - 1/5

3. Suna Choi, "Modeling and Design of BGA Type Package for low Inductuve Power/Ground Impedance and Simultaneous Switching Noise", M.S thesis,KAIST, Dec.2004, pp27-30.

4. Hiroshi Yoshida, Hiroshi Tsurumi, Yasuo Suzuki, "DC Offset Canceller in a Direct Conversion Receiver for QPSK Signal Reception", Personal, Indoor and Mobile Radio Communications, 1998, The Ninth IEEE International Symposium on Volume 3, 8-11 Sept. 1998 Page(s):1314 - 1318 vol.3 Digital Object Identifier 10.1109/PIMRC.1998.731406 